\title{
Unraveling the Composition and Behavior of Heterogeneous Lipid Nanodiscs by Mass Spectrometry
}

\author{
Kin Kuan Hoi, Carol V. Robinson, and Michael T. Marty ${ }^{\star}$ \\ Department of Chemistry, University of Oxford, Physical and Theoretical Chemistry Laboratory, \\ South Parks Road, OX1 3QZ (UK)
}

\begin{abstract}
Mass spectrometry (MS) has emerged as a powerful tool to study membrane protein complexes and protein-lipid interactions. Because they provide a precisely-defined lipid bilayer environment, lipoprotein Nanodiscs offer a promising cassette for membrane protein MS analysis. However, heterogeneous lipids create several potential challenges for native MS: additional spectral complexity, ambiguous assignments, and differing gas-phase behaviors. Here, we present strategies to address these challenges and streamline analysis of heterogeneous-lipid Nanodiscs. We show that using two lipids of similar mass limits the complexity of the spectra in heterogeneous Nanodiscs and that the lipid composition can be determined by using a dual Fourier transform approach to obtain the average lipid mass. Further, the relationship between gas-phase behavior, lipid composition, and instrumental polarity was investigated to determine the effects of lipid head group chemistry on Nanodisc dissociation mechanisms. These results provide unique mechanistic and methodological insights into characterization of complex and heterogeneous systems by mass spectrometry.
\end{abstract}

\section{Introduction}

Despite having important biochemical roles, membrane proteins remain challenging to study. Native or non-denaturing electrospray ionization (ESI) mass spectrometry (MS) has become a powerful approach to characterize membrane proteins, including protein-lipid complexes.1 Conventional methods rely on detergents to solubilize the membrane proteins and lipids prior to ionization.2-4 However, alternative membrane mimetics present unique opportunities for native MS analysis of lipid bilayer systems.5-7

Nanodiscs are self-assembly lipoprotein complexes consisting of a lipid bilayer encircled by two membrane scaffold proteins (MSP). 8 They provide a membrane mimetic with diameters ranging from 8 to $16 \mathrm{~nm}, 9$ enabling studies of membrane protein structure and interaction in native-like environments. 8,10 Because Nanodiscs are relatively monodisperse, they offer a promising platform for native MS. Initial native MS analysis of Nanodiscs without embedded membrane proteins demonstrated that they can be preserved intact and relatively

\footnotetext{
*Corresponding Author mtmarty@email.arizona.edu. Notes

The authors declare no competing financial interest.
} 
monodisperse in the gas-phase. 11 Recent work has utilized Nanodiscs with native MS for membrane protein studies. By reconstituting membrane proteins into Nanodiscs, intact membrane protein complexes can be delivered into the gas phase directly from the nativelike lipid bilayer environment.6 Using gradual collision induced dissociation (CID) of membrane protein-Nanodisc complexes, membrane protein oligomers can be released with a large number of bound lipids, which match the stoichiometries predicted in solution for the lipid annular belt and several subshells.7

Although prior native MS studies have generally used homogeneous Nanodiscs formed with a single lipid component, heterogeneous Nanodiscs can be formed with a precisely-defined lipid composition.12 Heterogeneous glycolipid Nanodiscs have been used to study the selectivity and binding kinetics of soluble protein-glycolipid interactions by native mass spectrometry.13-16 Although the heterogeneous Nanodisc ions appeared as an unresolved hump, the released protein-glycolipid complexes were well resolved.

The use of heterogeneous lipid Nanodiscs for MS has been restricted by several potential challenges that are common to complex molecular and macromolecular systems. First, heterogeneous lipids create greater polydispersity that complicates spectra. Second, additional mass possibilities can cause spectra to become more difficult to interpret and assign. Finally, the gas-phase behavior of Nanodiscs may change as the surface chemistry varies with different lipid compositions.

Here, we present strategies for overcoming these challenges and a framework for analysis of heterogeneous-lipid Nanodiscs that relies on average lipid mass as a reporter of lipid composition. Further, we explore the effects of lipid charge on gas-phase dissociation mechanisms of Nanodiscs under different instrumental polarities.

\section{Experimental Section}

Materials were purchased from Sigma Aldrich (St. Louis, MO) unless otherwise stated. Chromatography columns were manufactured by GE Healthcare (Piscataway, NJ). Powdered lipids, 1-palmitoyl-2-oleoyl-sn-glycero-3-phosphocholine (POPC), 1-palmitoyl-2-oleoyl-snglycero-3-phospho-(1'-rac-glycerol) (POPG), and 1-palmitoyl-2-oleoyl-sn-glycero-3phospho-L-serine (POPS) were purchased from Avanti Polar Lipids (Alabaster, AL). Lipids were dissolved in either Chloroform (POPC) or 1:5 methanol:chloroform (POPG and POPS) and stored at $-20{ }^{\circ} \mathrm{C}$. Lipid concentrations were determined by phosphate analysis. 17

MSP1D1(-) was obtained as previously described.7,9,18 Briefly, MSP1D1 was expressed in E. coli and purified by immobilized metal affinity chromatography (IMAC). The polyhistadine tag was cleaved with TEV protease to yield MSP1D1(-), which was then purified by reverse IMAC. MSP1D1(-) was concentrated to around $0.2 \mathrm{mM}$, flash frozen, and stored at $-80{ }^{\circ} \mathrm{C}$.

To study heterogeneous-lipid Nanodiscs in the gas phase, we prepared MSP1D1(-) Nanodiscs formed with POPG:POPC or POPS:POPC at compositions ranging from 0 to $100 \%$ at $25 \%$ intervals (Figure 1). Nanodiscs were prepared as described previously.11,19 Briefly, dissolved lipid stocks were dried under nitrogen gas flow and stored in a vacuum 
desiccator over-night. The dried lipid films were re-dissolved in two molar equivalent of 0.1 M sodium cholate. Different lipids were mixed as cholate stocks in appropriate quantities prior to reconstitution. MSP1D1(-) was thawed on ice and added to the cholate-dissolved lipids. The ratio of total lipid to MSP1D1(-) was 65:1. The reconstitution mixture was incubated on ice for $20 \mathrm{~min}$. Amberlite XAD-2 hydrophobic beads were added at roughly $1: 1 \mathrm{v} / \mathrm{v}$ ratio to initiate Nanodisc self-assembly. After overnight incubation, Nanodiscs were filtered and purified on a GE Healthcare Superose 6 Increase 10/300 size-exclusion chromatography (SEC) column equilibrated with $0.2 \mathrm{M}$ ammonium acetate (pH 6.8). Peak fractions were combined and concentrated to 5-25 $\mu \mathrm{M}$. Nanodiscs were flash frozen and stored at $-80{ }^{\circ} \mathrm{C}$ before use. In some cases, an additional centrifugal buffer exchange column (Micro Bio-Spin 6, Bio-Rad, Hercules, CA) equilibrated in $0.2 \mathrm{M}$ ammonium acetate (pH 6.8) was used to remove residual sodium salts when significant sodium adducts were observed in a mass spectrum. Three replicate Nanodisc samples were prepared at each lipid composition. Nanodiscs were characterized in solution by SEC using a Superdex 200 $3.2 / 300$ column as described previously. 7

MS analysis of Nanodiscs was performed using a modified Q-Exactive hybrid quadrupoleOrbitrap mass spectrometer (Thermo Fisher Scientific Inc., Bremen, Germany) optimized for analysis of high $\mathrm{m} / \mathrm{z}$ ions.20,21 Ionization was performed by nano-ESI, in both positive and negative polarity, using a static gold-coated capillary needle.22 Instrumental parameters were the same as previously described.7 MS in negative polarity used the same absolute values as positive polarity but with relevant voltages switched to the opposite polarity. CID was performed by increasing the voltage applied in the HCD cell from 0 to $190 \mathrm{~V}$ at $10 \mathrm{~V}$ steps with an acquisition time of two minutes per step. Measurements in both polarities were calibrated using cesium iodide.

The average masses of the lipids in Nanodiscs were measured from the spectra by a dual Fourier transform approach. The spectra were linearized by interpolating the spectrum at $0.01 \mathrm{Th}$ steps. The Nanodisc signal was isolated by first limiting the $\mathrm{m} / \mathrm{z}$ range to greater than $6500 \mathrm{~m} / z$. Then, the centroid of the Nanodisc signal was estimated by taking the maximum positions of the spectrum after smoothing with a broad Gaussian $(\sigma=1000 \mathrm{Th})$. The spectrum was multiplied by a Gaussian window centered at the centroid with $\sigma=1500$ Th as shown in Figure 2A. The dual Fourier transform approach applied two sequential fast Fourier transforms (FFT) to the processed mass spectra. Each FFT was performed using FFTPack in Scipy.23,24 Because the theoretical average lipid mass was between 749 and $762 \mathrm{Da}$ for all Nanodiscs, the experimental average lipid mass was measured as the maximum within 740 to $770 \mathrm{Da}$ in the second FFT. The dual Fourier transform method is built into UniDec,25 a Bayesian deconvolution program, which is available for download at unidec.chem.ox.ac.uk.

Nanodisc spectra are highly complex due to the overlapping lipid counts and charge state distributions. Because many of the ions are overlapped in $\mathrm{m} / z$ space, manual assignment is often impractical.26 To overcome this challenge we utilized UniDec to deconvolve the mass spectra (Figure S1).7,25 Raw MS files were converted into mzML format using ProteoWizard 27 and were then converted into text files by compressing the two minute acquisition using pymzml.28 The dual Fourier transform was performed prior to 
deconvolution to determine the average mass of lipids $m_{l}$ in the Nanodiscs. For deconvolution, spectra were subjected to Gaussian smoothing with $\sigma=5$ data points and curved background subtraction with a width of 100 . No linearization was performed. Deconvolution was performed with a mass range of 4-280 kDa and a charge range of 1-30. The peak shape was Gaussian with a 10 Th full-width at half-maximum. The Bayesian priors were smoothed using a Gaussian with $\sigma=0.5$ in the charge dimension and a log mean filter with a width of 3 in lipid count, where the mass of the oligomer used was the $m_{l}$ measured from each spectrum. Data were converted from $\mathrm{m} / \mathrm{z}$ to mass using interpolation at $10 \mathrm{Da}$ intervals. After deconvolution, ions overlapped in $\mathrm{m} / z$ space are separated in mass versus charge space (Figure S1E), which simplifies the interpretation of the MS data. Deconvolved distributions of both mass and charge (Figures S1B and S1D) were monitored as a function of collision voltage (Figures S1C and S1F). Mass defect analysis was performed as described previously7 using the $m_{l}$ values as the reference masses.

\section{Results and Discussion}

\section{Determining lipid composition in Nanodiscs}

Due to the stochastic nature of self-assembly, Nanodiscs have a distribution in the number of lipids per complex. Prior native MS studies have shown that homogeneous Nanodiscs have a polydispersity of around \pm 5 lipids per complex.26 Heterogeneous Nanodiscs have an additional dimension of polydispersity because each total lipid count can consist of multiple sub-states. For example, a complex with 125 total lipids formed with an average of 50\% POPG:POPC could exist in multiple distinct sub-states: 64:61, 63:62, 62:63, 61:64, and so forth, which we predict would be populated according to a binomial distribution. This additional dimension of polydispersity presents a challenge for native MS because additional states may complicate the spectra and lower the signal-to-noise ratio.

To limit the complexity of the mass spectra, we used heterogeneous-lipid Nanodiscs with two synthetic lipids of slightly different mass. Nanodiscs were prepared with POPG:POPC or POPS:POPC at five different compositions (Figure 1). POPG and POPC are $11 \mathrm{Da}$ different while POPS and POPC are only 2 Da apart. Because the lipids are similar in mass, sub-states are overlapped in the spectrum as shown in Figure 1. Thus, the native mass spectrum shows a single peak at 125 lipids per complex for a number of distinct sub-states, such as 63:62 and 62:63.

By compressing the sub-states into a single peak, we solve the complexity problem, but the lost information creates an additional challenge of interpretation. In the most extreme case of isobaric lipids, we could measure the total stoichiometry of the lipids, but it would be impossible to determine the relative composition of individual lipids from the masses alone. For lipids of different mass, unambiguous interpretation and assignment of spectra requires determination of the average lipid composition.

Although the average lipid composition is difficult to measure directly from a single mass, the average lipid mass, $m_{l}$, can be determined from the distance between peaks, such as between 125 and 126 lipids per complex. Our initial approach to determining the average lipid mass was to measure the spacing between peaks in deconvolved zero-charge mass 
spectra (Figure S1D), either through averaging the difference between peaks or using a more sophisticated autocorrelation approach. However, the precision of both approaches was lacking and sensitive to deconvolution parameters.

Therefore, we sought an approach to measure the spacing between peaks directly from the mass spectra. Each mass spectrum contains multiple masses divided by several different charges. For a given charge state, $z$, a distribution in the total number of lipids per complex will produce a regularly-spaced series of peaks in the mass spectrum, each separated by $m_{l} / z$. Because these regularly-spaced peaks have a characteristic frequency, we reasoned that Fourier transforms would be suitable for extracting information from the spectra. A Fourier transform of a Nanodisc mass spectrum results in a primary frequency at $z / m_{l}$ in the reciprocal space, $v$, where the units are $\mathrm{Th}^{-1}$ (Figure 2B). As Nanodisc ions exist in several consecutive charge states, the Fourier transform of the mass spectrum leads to frequency peaks at $\left(z / m_{l},(z+1) / m_{l},(z+2) / m_{l}, \ldots\right)$ that each differs by $1 / m_{l}$.

Similarly, because the frequency peaks in $v$ space are regularly spaced by $1 / m_{1}$, we can perform a second Fourier transform. The Fourier transform of $v$ space gives a primary frequency at $m_{l}$ in $v(v)$ space (Figure $2 \mathrm{C}$ ). Thus, we can directly measure the average lipid mass of all charge states using a dual Fourier transform. Because the charge is unity at the primary frequency in $v(v)$ space, the units become Da rather than Th. Moreover, $v(v)$ space will be sampled at the same density as the original spectrum, which was linearized at 0.01 Th. We determine the average mass of lipids inside the Nanodisc as the primary frequency in the second Fourier transform, which is the maximum between 740 and $770 \mathrm{Da}$ (Figure 2D). Interestingly, the width of the frequency peak is greatest for Nanodiscs with 50\% POPG:POPC (Figure 2D), likely due to broader peaks from the binomial distribution of microstates.

The dual Fourier transform approach assumes a uniform $m_{l}$. Although this is true on a local scale for consecutive charge states, we observe three potentially non-uniform species in some spectra: the Nanodisc, which may be depleted of lipids by dissociation, and the dissociation products, MSP and free lipids. For this study, we considered only the region of the spectra containing MSP and Nanodiscs. To determine the average lipid mass from each species separately, we applied a sliding Gaussian window (Figure 2A) to measure the dual Fourier transform as a function of $m / z$, as shown in Figures 3B-D. The overall average lipid mass was determined from the window centered at the estimated centroid of the mass spectrum.

Using the dual Fourier transform approach, we can measure the average lipid mass directly from the raw data with minimal processing and assumptions. Comparing the average of spectra collected from 60 to $100 \mathrm{~V}$ CID for three replicate samples, the average lipid mass is very precise, showing a standard deviation of less than $0.3 \mathrm{Da}$ and generally falling within a standard deviation of the theoretical average lipid mass (Figure 2E).

The relative composition of the two lipids in the Nanodisc was interpolated from the average lipid mass. Because POPC and POPG differ by $11 \mathrm{Da}$, the relative composition can be interpolated precisely based on the average lipid mass to a relative standard deviation of 
around 3\%. As shown in Figure 2F, the measured lipid composition agrees closely with the expected input lipid content. Thus, the lipid composition remains the same before and after Nanodisc self-assembly.12 Although POPS:POPC Nanodiscs show a precise average mass, the small mass difference (2 Da) between POPS and POPC translates into a larger error in interpolated composition of around $15 \%$. To precisely determine the relative composition of POPS to POPC at this instrumental resolution, isotopic labelling would be necessary to increase the mass difference.

Having established a means of determining the lipid composition, we investigated how composition is effected by collisional dissociation. By monitoring the average lipid mass for POPG:POPC Nanodiscs from 60 to $190 \mathrm{~V}$, we observed significant changes in the lipid composition at elevated collision energies (Figure 3A). Interestingly, the average lipid masses of Nanodiscs with POPC and POPG first decrease at medium collision voltage but then increase at high collision voltage. At medium collision voltage, POPC is preferentially ejected, leading to an enrichment of POPG and therefore a decreased average lipid mass in the lipid-depleted Nanodiscs (Figure 3C). At high collision voltage, Nanodiscs are largely fragmented. MSP dimer and monomer become the strongest signals in the spectrum. Thus, the centroid of the spectrum shifts toward MSP. Ejected MSP carries a small number of lipids with a preference for POPC, which causes an overall increase in the average lipid mass as the intensity of the MSP signal overtakes the Nanodisc signal (Figure 3D).

\section{Effects of polarity on Nanodisc dissociation}

Given that there is a preference for dissociation of certain lipids at elevated collision energies, we reasoned that lipid charge may play a significant role in dissociation mechanisms. Nanodiscs have been shown to dissociate by continuously ejecting lipids as the activation energy increases. $26 \mathrm{We}$ observed a similar phenomenon for all heterogeneous Nanodiscs as shown in Figure S2 and S3. However, homogeneous Nanodiscs containing POPG or POPS showed a prolonged stability followed by a discontinuous fragmentation into MSP monomers (Figure 4).

To explore the relationship between dissociation behavior, lipid composition, and instrumental polarity, we repeated the experiments in negative polarity. In negative polarity, a continuous dissociation pattern is observed for homogenous Nanodiscs containing POPG and POPS (Figure 4 and S3) as well as all heterogeneous Nanodiscs (Figures S2 and S3). However, homogeneous POPC Nanodiscs retain a constant mass and charge throughout all collision energies, exhibiting an extraordinary stability in negative polarity similar to that of homogeneous POPG and POPS Nanodiscs in positive polarity (Figure 4 and S3).

To understand this polarity dependence, we considered the charge properties for each lipid as observed both for $\mathrm{p} K$ a values in solution lipid bilayers29,30 as well as their ESI behavior from lipidomics experiments.31,32 All three lipids share the same hydrocarbon tail but different head groups. Structures of the head group at neutral charge are shown in Figure 1. POPC can hold either a net neutral or a +1 charge. It cannot hold a negative charge due to the fixed positive charge on the quaternary amine. 33 Thus, in the absence of adducts, POPC ionizes in positive polarity but will be neutral in negative polarity, where POPC Nanodiscs exhibit unusual stability. 
On the other hand, POPG can hold either a neutral or a -1 charge. It cannot hold a positive charge and will thus ionize in negative mode. Similarly, although POPS can possess from +1 to -2 charges, it will only be positively charged at very low $\mathrm{pH}$ and is anionic at neutral $\mathrm{pH}$. Thus, both POPG and POPS ionize preferentially in negative mode and will be neutral in positive polarity, where homogeneous Nanodiscs of these lipids are stable to continuous dissociation. Therefore, as observed in both positive and negative polarities, homogeneous Nanodiscs possess a special stability under the instrumental polarity where the lipids are neutral, demonstrating a polarity dependent dissociation pattern that is controlled by the chemistry of the lipid head group.

However, charge is not the sole factor controlling dissociation. In both polarities, heterogeneous Nanodiscs undergo continuous dissociation (Figure S2 and S3), as seen with homogeneous POPC Nanodiscs in positive polarity and POPG and POPS in negative polarity. Although some preference is observed at higher collision energies as shown above, this continuous dissociation is not initially selective of only the charged lipid. Thus, at low collision voltage, lipids are ejected from the Nanodisc in both the charged and neutral states. Neutral ejection can also be observed for homogeneous Nanodiscs in the appropriate polarity because the decrease in lipid stoichiometry upon activation is larger than the corresponding decrease in charge. Thus, charged lipids are required for continuous dissociation, but not all lipids that dissociate are charged.

In many cases, we observe a bimodal distribution of charge and/or mass (Figure S2 and S3). Using mass defect analysis as described previously, 7 we find that masses between 70 and $150 \mathrm{kDa}$ contain $2 \mathrm{MSP}$ belts, as expected for Nanodiscs in solution. At elevated collision voltage, peaks are observed below $60 \mathrm{kDa}$ that correspond to dissociation products containing a single MSP belt and a number of lipids as seen previously.7 Interestingly, for Nanodiscs containing a high percentage of anionic lipids, peaks are observed above $150 \mathrm{kDa}$ that correspond to lipoprotein complexes containing a large number of lipids and three MSP belts. While we cannot rule out the possibility of a gas-phase or ESI artifact, SEC analysis reveals that Nanodiscs become more polydisperse above 50\% anionic lipid content (Figure S4). This suggests that some of the increased polydispersity manifested as multiple distributions in the mass spectra may be due to solution-phase artifacts.

However, POPC Nanodiscs, which are monodisperse in solution measurements and in positive polarity, show a distinct bimodal mass distribution in negative polarity with a mass difference of about $30 \mathrm{kDa}$ between the two distributions. Because these Nanodiscs are otherwise monodisperse, this is likely a gas-phase or ESI artifact. The mass defect for MSP1D1(-) with respect to POPC is nearly zero,7 so we cannot determine from the mass alone whether the second distribution resulted from the loss of MSP or lipids. However, it is likely due to the loss of lipids rather than MSP because similar bimodal distributions in positive mode for heterogeneous Nanodiscs definitively show two belts present for species from 70 to $150 \mathrm{kDa}$. This suggests that the multimodal mass distribution is due to concerted ejection of a large number of lipids. Interestingly, concerted dissociation appears to occur with little or no loss of charge, suggesting the dissociation product may be due primarily to neutral loss. Also, unlike continuous dissociation, it seems to be independent of collision 
voltage. Although the mechanisms of this concerted ejection are unknown, it is clearly dependent on lipid and instrumental polarity.

\section{Conclusion}

We have demonstrated a framework for analysis of lipoprotein Nanodiscs containing a heterogeneous population of lipids. To limit complexity of the spectra, we utilized a binary combination of lipids of similar mass. By using a dual Fourier transform approach, we are able to accurately measure the average lipid mass of a Nanodisc by native MS with high precision. The average lipid mass was used to interpolate the lipid composition of these binary mixtures of lipids, which clearly demonstrated that Nanodiscs can be formed with a precise lipid composition. CID experiments revealed the effects of lipid and instrumental polarity on the gas-phase dissociation of Nanodiscs. We found that having a chargeable lipid is required for continuous dissociation, but that not all dissociated lipids are ejected as charged species. These results provide a unique perspective on dissociation mechanisms of protein-lipid ions and set the stage for native MS analysis of more complex and heterogeneous Nanodisc complexes with embedded membrane proteins. Moreover, this approach will enable mass spectrometry characterization of complex and heterogeneous mixtures, including biological and synthetic polymers.

\section{Supporting Information}

Refer to Web version on PubMed Central for supplementary material.

\section{Acknowledgment}

MTM is funded by program grant G1000819 from the Medical Research Council. CVR is funded by European Research Council Investigator Award (IMPRESSS, grant no. 268851).

\section{References}

(1). Bechara C, Robinson CV. J Am Chem Soc. 2015; 137:5240-5247. [PubMed: 25860341]

(2). Barrera NP, Di Bartolo N, Booth PJ, Robinson CV. Science. 2008; 321:243-246. [PubMed: 18556516]

(3). Laganowsky A, Reading E, Hopper JTS, Robinson CV. Nat Protoc. 2013; 8:639-651. [PubMed: 23471109]

(4). Barrera NP, Robinson CV. Annu Rev Biochem. 2011; 80:247-271. [PubMed: 21548785]

(5). Calabrese AN, Watkinson TG, Henderson PJF, Radford SE, Ashcroft AE. Anal Chem. 2015; 87:1118-1126. [PubMed: 25495802]

(6). Hopper JT, Yu YT, Li D, Raymond A, Bostock M, Liko I, Mikhailov V, Laganowsky A, Benesch JL, Caffrey M, Nietlispach D, et al. Nat Methods. 2013; 10:1206-1208. [PubMed: 24122040]

(7). Marty MT, Hoi KK, Gault J, Robinson CV. Angew Chem Int Ed. 2016; 55:550-554.

(8). Bayburt TH, Sligar SG. FEBS Lett. 2010; 584:1721-1727. [PubMed: 19836392]

(9). Ritchie, TK., Grinkova, YV., Bayburt, TH., Denisov, IG., Zolnerciks, JK., Atkins, WM., Sligar, SG. Methods Enzymol. Nejat, D., editor. Vol. 464. Academic Press; New York: 2009. p. 211-231.

(10). Schuler, M., Denisov, I., Sligar, S. Lipid-Protein Interactions. Kleinschmidt, JH., editor. Vol. 974. Humana Press; 2013. p. 415-433.

(11). Marty MT, Zhang H, Cui W, Blankenship RE, Gross ML, Sligar SG. Anal Chem. 2012; 84:89578960. [PubMed: 23061736] 
(12). Shaw AW, Pureza VS, Sligar SG, Morrissey JH. J Biol Chem. 2007; 282:6556-6563. [PubMed: 17200119]

(13). Leney AC, Fan X, Kitova EN, Klassen JS. Anal Chem. 2014; 86:5271-5277. [PubMed: 24779922]

(14). Han L, Kitova EN, Li J, Nikjah S, Lin H, Pluvinage B, Boraston AB, Klassen JS. Anal Chem. 2015; 87:4888-4896. [PubMed: 25859741]

(15). Leney AC, Rezaei Darestani R, Li J, Nikjah S, Kitova EN, Zou C, Cairo CW, Xiong ZJ, Privé GG, Klassen JS. Anal Chem. 2015; 87:4402-4408. [PubMed: 25803566]

(16). Zhang Y, Liu L, Daneshfar R, Kitova EN, Li C, Jia F, Cairo CW, Klassen JS. Anal Chem. 2012; 84:7618-7621. [PubMed: 22920193]

(17). Chen PS, Toribara TY, Warner H. Anal Chem. 1956; 28:1756-1758.

(18). Denisov IG, Grinkova YV, Lazarides AA, Sligar SG. J Am Soc Mass Spectrom. 2004; 126:34773487.

(19). Bayburt TH, Grinkova YV, Sligar SG. Nano Lett. 2002; 2:853-856.

(20). Dyachenko A, Wang G, Belov M, Makarov A, de Jong RN, van den Bremer ETJ, Parren PWHI, Heck AJR. Anal Chem. 2015; 87:6095-6102. [PubMed: 25978613]

(21). Gault J, Donlan JA, Liko I, Hopper JT, Gupta K, Housden NG, Struwe WB, Marty MT Mize T, Bechara C, Zhu Y, et al. Nat Methods. 2016; 13:333-336. [PubMed: 26901650]

(22). Hernandez H, Robinson CV. Nat Protoc. 2007; 2:715-726. [PubMed: 17406634]

(23). Oliphant TE. Comput Sci Eng. 2007; 9:10-20.

(24). Millman KJ, Aivazis M. Comput Sci Eng. 2011; 13:9-12.

(25). Marty MT, Baldwin AJ, Marklund EG, Hochberg GKA, Benesch JLP, Robinson CV. Anal Chem. 2015; 87:4370-4376. [PubMed: 25799115]

(26). Marty M, Zhang H, Cui W, Gross M, Sligar S. J Am Soc Mass Spectrom. 2014; 25:269-277. [PubMed: 24353133]

(27). Chambers MC, Maclean B, Burke R, Amodei D, Ruderman DL, Neumann S, Gatto L, Fischer B, Pratt B, Egertson J, Hoff K, et al. Nat Biotechnol. 2012; 30:918-920. [PubMed: 23051804]

(28). Bald T, Barth J, Niehues A, Specht M, Hippler M, Fufezan C. Bioinformatics. 2012; 28:10521053. [PubMed: 22302572]

(29). Tsui FC, Ojcius DM, Hubbell WL. Biophys J. 1986; 49:459-468. [PubMed: 3955180]

(30). Marsh, D. Handbook of Lipid Bilayers. 2nd ed. CRC Press; Boca Raton, FL: 2013. p. 43-860.

(31). Brügger B, Erben G, Sandhoff R, Wieland FT, Lehmann WD. Proc Natl Acad Sci U S A. 1997; 94:2339-2344. [PubMed: 9122196]

(32). Ivanova PT, Cerda BA, Horn DM, Cohen JS, McLafferty FW, Brown HA. Proc Natl Acad Sci U S A. 2001; 98:7152-7157. [PubMed: 11416200]

(33). Godzien J, Ciborowski M, Martínez-Alcázar MP, Samczuk P, Kretowski A, Barbas C. J Proteome Res. 2015; 14:3204-3216. [PubMed: 26080858] 
A

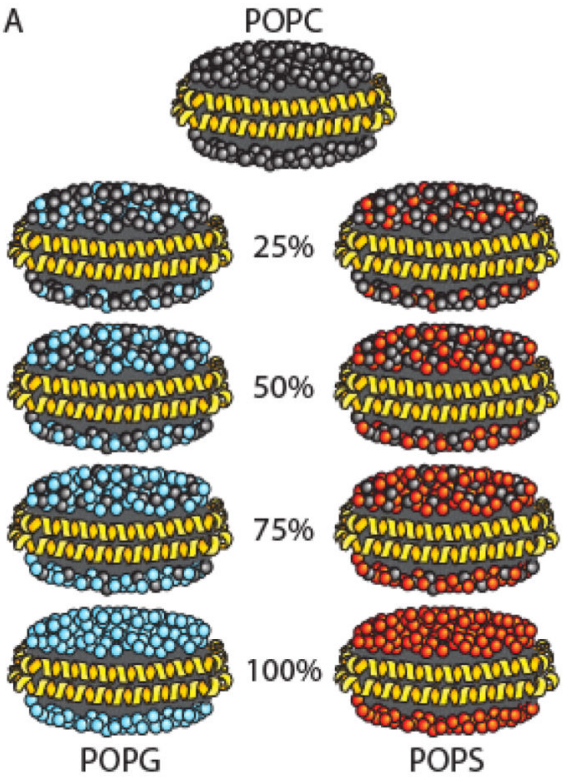

POPC: $760 \mathrm{Da}$<smiles>C[N+](C)(C)CCOP(=O)([O-])O[Na]</smiles>

POPG: $749 \mathrm{Da}$<smiles>[Y]OP(=O)(O)OCC(O)CO</smiles>

POPS: $762 \mathrm{Da}$<smiles>[CH]OP(=O)([O-])OC[C@H]([NH3+])C(=O)O</smiles>

B

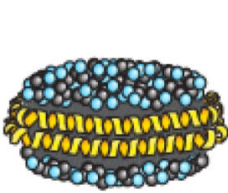

63:62 $62: 63$

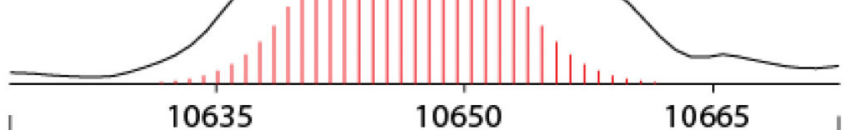

I
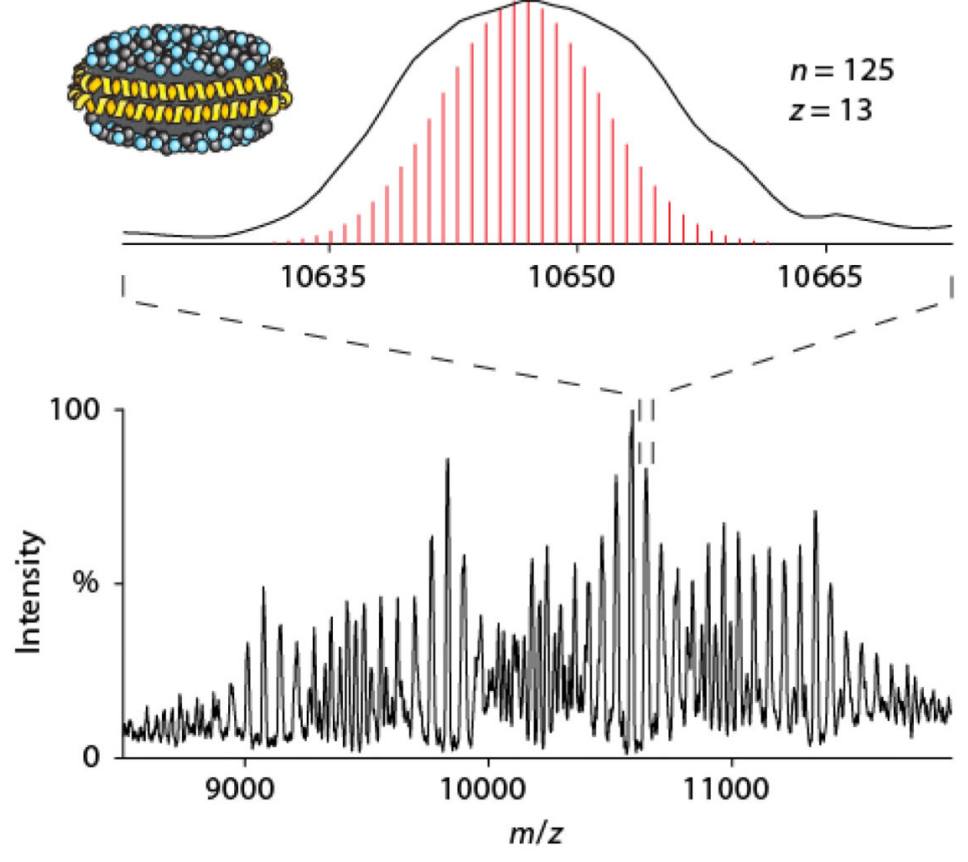

Figure 1.

Schematic of Nanodiscs with a binary combination of lipids and illustration of Nanodisc sub-states arising from different lipid stoichiometries. (A) MSP1D1(-) (yellow) Nanodiscs were prepared with pure or binary combinations of POPC (black), POPG (blue), and POPS (red). Masses and head group structures of neutral lipids are shown on the right. (B) The raw mass spectrum of 50\% POPG:POPC Nanodiscs was collected at $80 \mathrm{~V} \mathrm{CID} \mathrm{(black).} \mathrm{The}$ zoomed region corresponds primarily to 125 lipids $(n)$ per complex at a charge state of +13 $(z)$. The red bars denote the theoretical binomial distribution of sub-states arising from the 
same total number of lipids but different combinations of POPG and POPC (e.g. 63:62 and 62:63). 

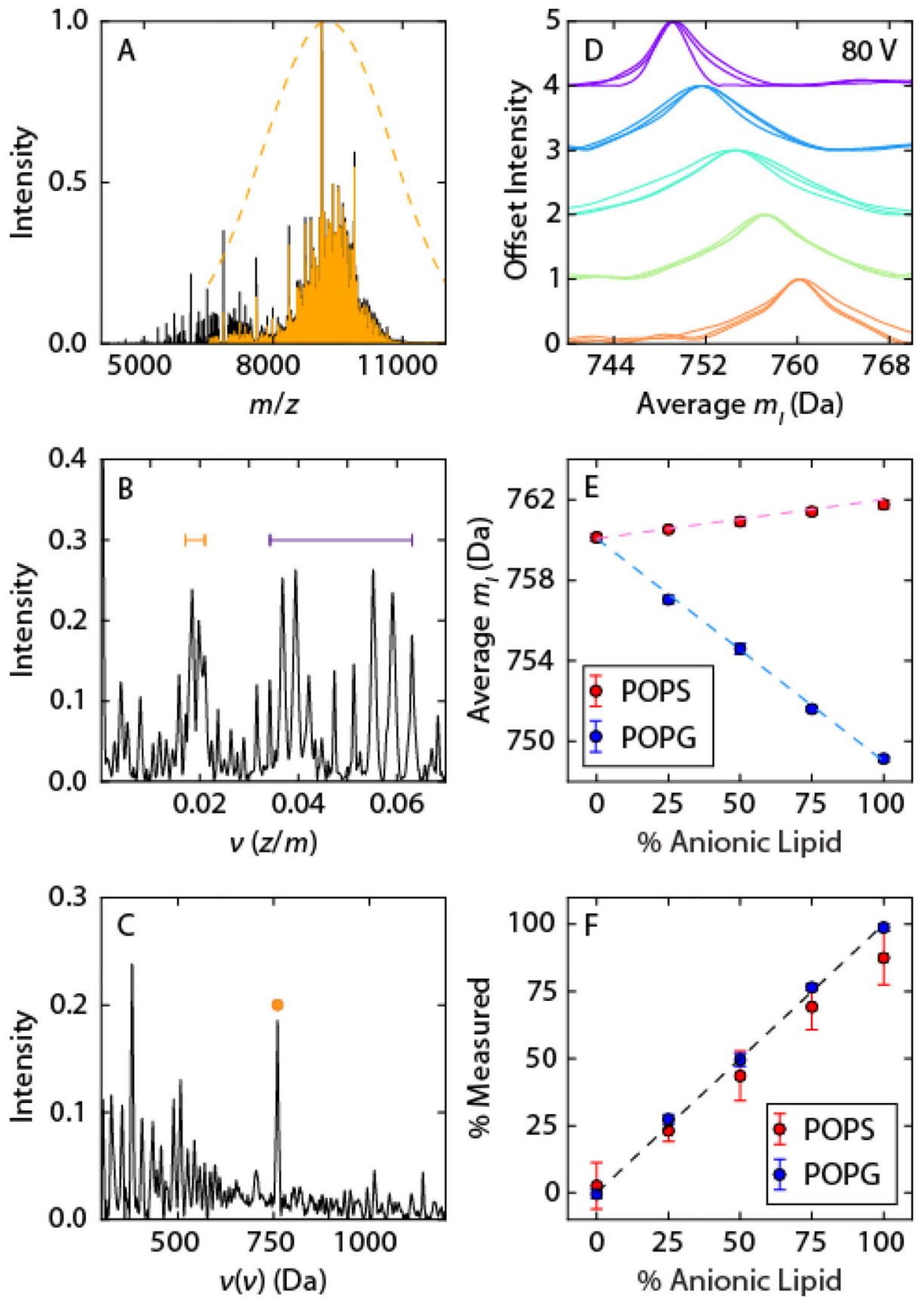

Figure 2.

Measuring average lipid mass $\left(m_{l}\right)$ of Nanodiscs by dual Fourier transform. (A) The raw spectrum of POPC Nanodisc at $80 \mathrm{~V}$ (black) was multiplied with a Gaussian window (orange dashed) to yield a windowed spectrum (orange). (B) A Fourier transform of the windowed spectrum yielded primary frequency peaks (orange bar) for Nanodiscs with different change states as well as harmonic peaks (purple bar) in $v$ space. (C) The second Fourier transform gives a peak at $m_{l}$ (orange $d o t$ ) in $v(v)$ space. (D) As POPG content increases from 0 to $100 \%$ (orange to purple), a corresponding shift is observed in the peak in 
$v(v)$ space of replicate POPG:POPC spectra collected at $80 \mathrm{~V}$. (E) The measured $m_{l}$ of POPG:POPC (blue) and POPS:POPC (red) Nanodiscs averaged from the mass spectra collected at 60 to $100 \mathrm{~V}$ over 3 replicates agree within one standard deviation (error bars) of the theoretical $m_{l}$ (dashed). (F) The corresponding lipid composition interpolated from $\mathrm{E}$ agree with the theoretical values (dashed). 

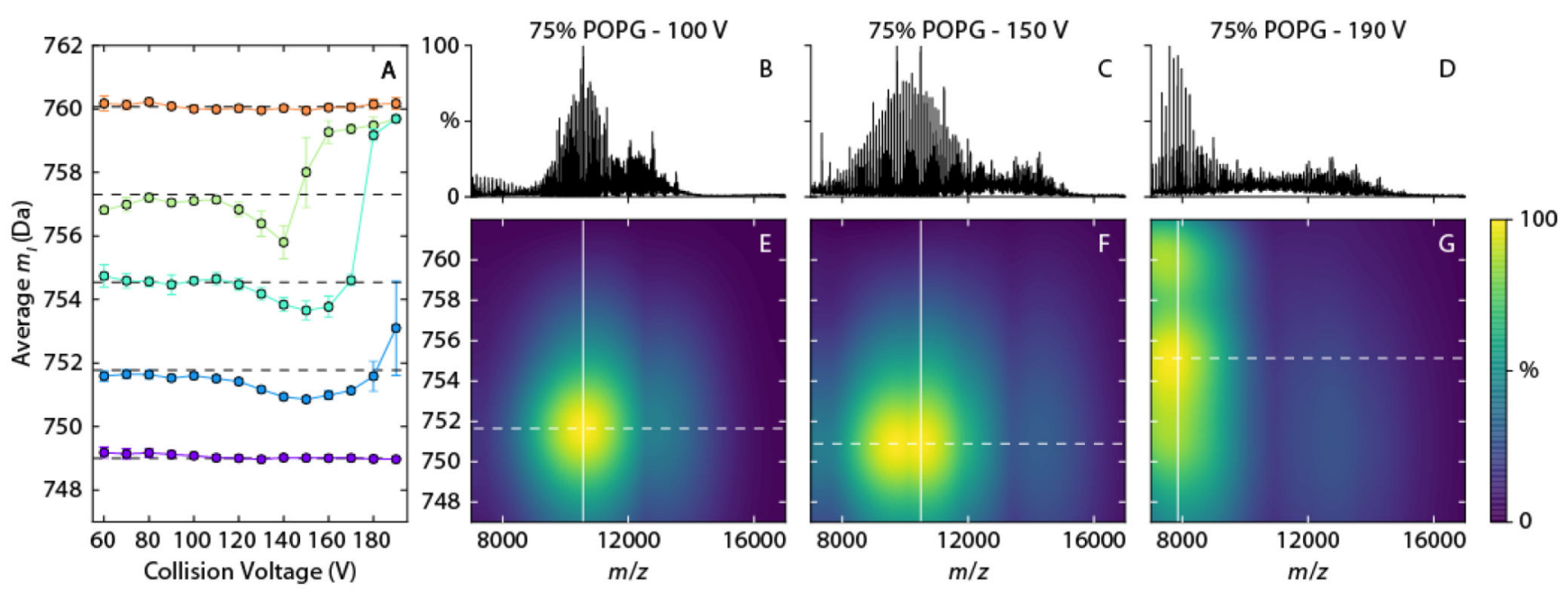

Figure 3.

Average lipid mass $\left(m_{l}\right)$ of Nanodiscs. (A) $m_{l}$ of Nanodiscs with POPG:POPC compositions ranging from 0 to $100 \%$ (orange to purple) is shown as the mean and standard deviation of three replicate samples at 60 to 190 V CID. Dashed lines denote the theoretical $m_{l}$ for Nanodiscs at each composition. Representative spectra from 75\% POPG Nanodiscs at (B) $100 \mathrm{~V}$, (C) $150 \mathrm{~V}$, and (D) $190 \mathrm{~V}$ CID are shown. The corresponding plots (E), (F) and (G) show the average lipid mass determined from the dual FFT (ordinate) as a function of the Gaussian window position (abscissa). The intensity is normalized by the maximum intensity of the smoothed mass spectrum. The average $m_{l}$ is measured as the position of the maximum peak in $v(v)$ space (horizontal dashed line) when the center of the Gaussian window aligns with the estimated centroid (vertical lines). 

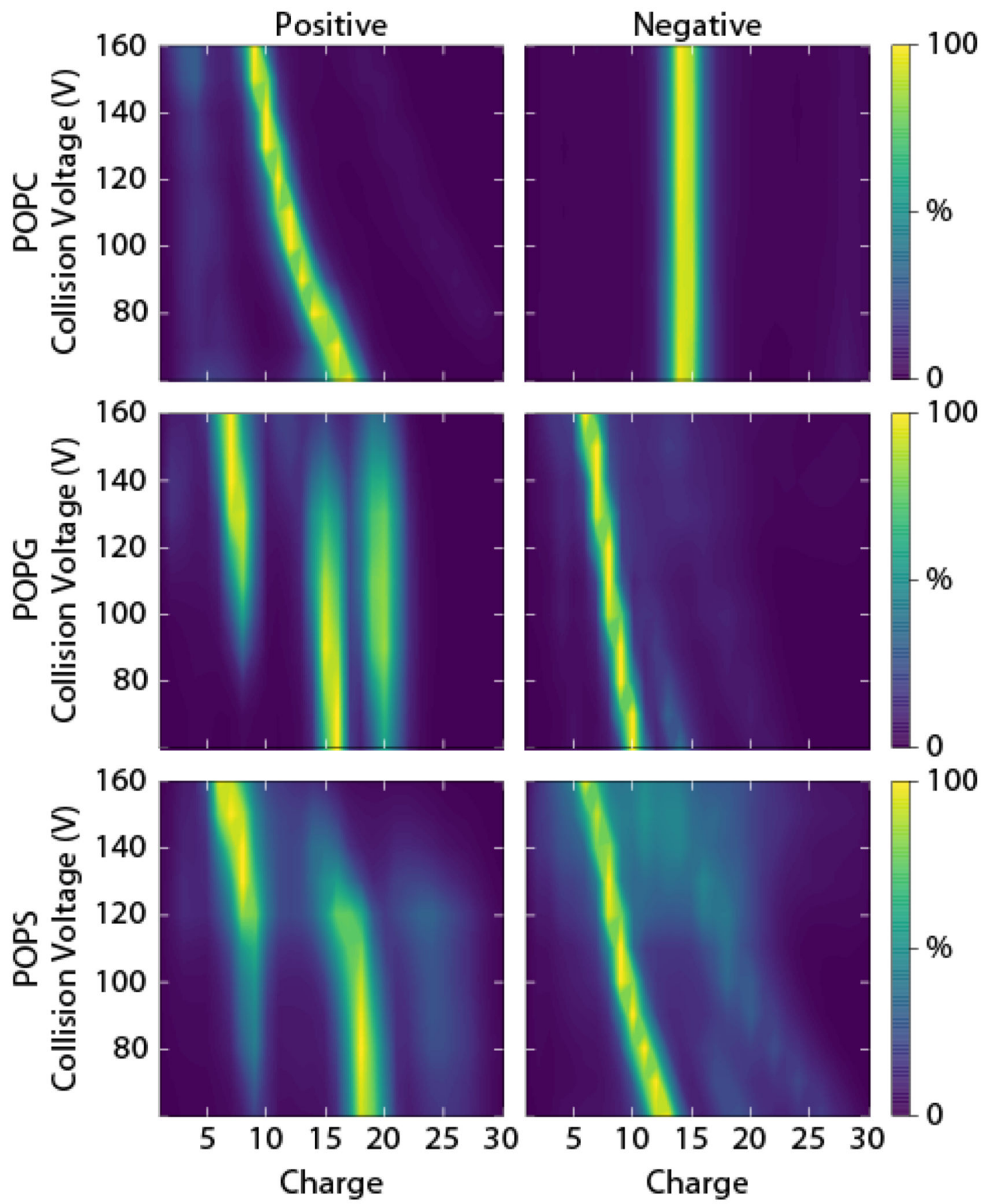

Figure 4.

The total charge distributions of homogeneous Nanodiscs as a function of collision voltage for POPC (top), POPG (middle), and POPS (bottom) Nanodiscs in positive (left) and negative (right) polarity. 